\title{
Repositórios de Objetos de Aprendizagem: uma análise comparativa com ênfase no reúso de conteúdos
}

\author{
Rossana de Paula Junqueira ${ }^{1,2,3}$, Bernadette Farias Lóscio ${ }^{1}$ \\ ${ }^{1}$ Centro de Informática - Universidade Federal de Pernambuco (UFPE) \\ Caixa Postal 7851 - 50.732-970 - Recife - PE - Brasil \\ ${ }^{2}$ Instituto Federal do Sertão Pernambucano (IFSertão-PE) - Campus Petrolina \\ BR 407, KM 08 - Jardim São Paulo - 56.314-520 - Petrolina - PE - Brasil \\ ${ }^{3}$ Faculdade de Ciências Aplicadas e Sociais de Petrolina (FACAPE) \\ Campus Universitário s/n - Vila Eduardo - 56.328-903 - Petrolina - PE - Brasil \\ rpj@cin.ufpe.br,bfl@cin.ufpe.br

\begin{abstract}
One of the key issues in using Learning Objects (LOs) is their reusability, i.e., a single learning object may be used in multiple contexts for multiple purposes. Currently, there are several learning objects repositories available on the Web, which provide means for the storage and retrieval of LOs. These repositories make easier the access to LOs, however they still have several limitations when it comes to the reuse of educational contents. In this context, this paper presents a comparative analysis between various repositories of LOs focusing on the content reuse. From this analysis, we aim to identify the requirements for new proposals for organization and sharing of learning objects on the Web that promote and facilitate the reuse of educational contents.
\end{abstract} \\ Resumo. Uma das principais características dos Objetos de Aprendizagem \\ (OA) é a sua reutilização, ou seja, um OA pode ser usado em diferentes \\ contextos e por usuários diversos. Atualmente, existem diversos repositórios \\ de Objetos de Aprendizagem disponíveis na Web, os quais oferecem meios \\ para o armazenamento e recuperação de OAs. Apesar de facilitarem o acesso \\ aos OAs, os repositórios existentes ainda possuem diversas limitações quando \\ se trata da reutilização de conteúdos educacionais. Neste contexto, este artigo \\ apresenta uma análise comparativa entre diversos repositórios de OAs, tendo \\ como ênfase o reúso dos conteúdos. A partir desta análise, espera-se \\ identificar os requisitos necessários para novas propostas de organização e \\ compartilhamento de objetos de aprendizagem na Web que promovam e \\ facilitem o reúso de conteúdos educacionais.
}

\section{INTRODUÇÃO}

Com o intuito de facilitar o compartilhamento de conteúdos educacionais disponíveis na Web, surgem novas soluções, como as baseadas em Objetos de Aprendizagem (OAs). Podemos citar como exemplos de OAs: vídeos, imagens, hipertextos, apresentação de slides, livros e apostilas [Wiley, 2000]. A partir do momento em que os OAs são pensados como recursos que possam ser adaptados, para serem reutilizados em situações diferentes e alterados por outros usuários, eles podem ser considerados um 
Recurso Educacional Aberto (REA). Estes recursos devem estar sob domínio público ou possuírem licenças de direito autoral livre. O termo REA engloba o conceito de objeto de aprendizagem incluindo direitos de uso, reúso, revisão, remix e redistribuição [Amiel et al., 2010].

Neste contexto, este artigo apresenta os resultados de um estudo comparativo entre repositórios de OAs disponíveis na Web. A análise comparativa contemplou 48 repositórios e buscou fazer uma avaliação a partir da disponibilização dos objetos de aprendizagem como recursos educacionais abertos. Na avaliação dos repositórios, foram consideradas características inerentes aos Objetos de Aprendizagem, bem como características de Dados Abertos, as quais foram consideradas relevantes por tratar aspectos relacionados ao uso e reúso de conteúdos.

O restante deste artigo esta organizado da seguinte forma: a Seção 2 apresenta os conceitos básicos sobre Objetos de Aprendizagem, Dados Abertos, Recursos Educacionais Abertos e Repositórios de Objetos de Aprendizagem, a Seção 3 discute a análise comparativa dos repositórios de objetos de aprendizagem, e a Seção 4 apresenta as considerações finais.

\section{CONCEITOS BÁSICOS}

Nesta seção, apresentamos alguns conceitos relevantes para o entendimento do estudo comparativo proposto neste trabalho.

\subsection{Objetos de Aprendizagem}

Os objetos de aprendizagem são ferramentas ou recursos digitais que podem ser utilizados e reutilizados no suporte ao aprendizado. Apesar de ser um conceito bastante difundido, não existe um consenso quanto à definição do termo $\mathrm{OA}$, de tal forma que suas principais características foram definidas por diversos autores. O Quadro 1 apresenta algumas dessas características com os seus respectivos autores,

Quadro 1: Características Técnicas dos OAs

\begin{tabular}{|c|c|c|c|}
\hline $\mathbf{N}^{0}$ & Característica & Definição & Autor \\
\hline 1 & Reusabilidade & $\begin{array}{l}\text { Possibilidade de incorporar o OA em múltiplas aplicações e para } \\
\text { diferentes propósitos, não exclusivamente para o qual foi concebido. }\end{array}$ & $\begin{array}{l}\text { [Wiley, } \\
\text { 2000] }\end{array}$ \\
\hline 2 & Interoperabilidade & $\begin{array}{l}\text { No contexto de OAs, implica na possibilidade dos objetos serem } \\
\text { utilizados em qualquer plataforma, repositório, sistema operacional, } \\
\text { navegador Web e sistema de gerenciamento de conteúdos. }\end{array}$ & $\begin{array}{l}\text { [Silva \& } \\
\text { Santanchè, } \\
\text { 2008] }\end{array}$ \\
\hline 3 & Granularidade & $\begin{array}{l}\text { É a característica que evidência o fato de que um objeto pode ser } \\
\text { agrupado em conjuntos maiores de conteúdos. }\end{array}$ & $\begin{array}{l}\text { [Tarouco, } \\
2010]\end{array}$ \\
\hline 4 & Autonomia & Diz respeito ao fato dos OAs poderem ser apresentados individualmente. & [Silva, 2004] \\
\hline 5 & Customização & $\begin{array}{l}\text { Como os objetos são independentes, a ideia de utilização dos mesmos em } \\
\text { um curso ou em vários cursos ao mesmo tempo torna-se real, e cada } \\
\text { instituição educacional pode utilizar os objetos e combiná-los da maneira } \\
\text { mais adequada. }\end{array}$ & $\begin{array}{l}\text { [Macêdo et. } \\
\text { al., 2007] }\end{array}$ \\
\hline 6 & Escalabilidade & $\begin{array}{l}\text { Esta característica está relacionada à facilidade da utilização do OA com } \\
\text { um pequeno ou grande número de pessoas. }\end{array}$ & [Silva, 2004] \\
\hline
\end{tabular}

Uma das discussões referente aos OAs diz respeito ao seu nível de fragmentação [Wiley, 2000]. A decisão sobre a fragmentação deve ser equilibrada entre os benefícios da reutilização e os gastos com a catalogação. Quanto menor a granularidade, maiores os gastos com a catalogação (metadados), mas também maior será a reutilização do OA. 
A fragmentação permite a construção de pequenos componentes instrucionais que podem ser reutilizados várias vezes em diferentes contextos.

\subsection{Dados Abertos e Recursos Educacionais Abertos}

O conceito de Dado Aberto é bastante amplo e, segundo a Open Knowledge Foundation ${ }^{l}$, consiste em qualquer dado que pode ser livremente utilizado, reutilizado e redistribuído por qualquer um. Dados Abertos são publicados e compartilhados na Web em formatos legíveis por máquinas e que podem ser novamente usados de forma livre pela sociedade. Um dado é considerado aberto quando apresenta as características exibidas no Quadro 2.

Quadro 2: Características dos Dados Abertos

\begin{tabular}{|c|l|l|}
\hline $\mathbf{N}^{0}$ & \multicolumn{1}{|c|}{ Característica } & \multicolumn{1}{c|}{ Conceito } \\
\hline 1 & Disponibilidade e acesso & $\begin{array}{l}\text { O dado precisa estar disponível por inteiro. Deve estar num formato } \\
\text { conveniente e modificável. }\end{array}$ \\
\hline 2 & Reúso e redistribuição & $\begin{array}{l}\text { O dado precisa ser fornecido em condições de reúso e redistribuição podendo } \\
\text { ser combinado com outros. }\end{array}$ \\
\hline 3 & Participação universal & $\begin{array}{l}\text { Todos podem usar, reusar e redistribuir o dado sem restrições de áreas, } \\
\text { pessoas ou grupos. }\end{array}$ \\
\hline
\end{tabular}

Por outro lado, o conceito de Recurso Educacional Aberto, ou simplesmente REA, tem um escopo mais restrito. REA diz respeito à provisão de recursos educacionais fazendo uso de ferramentas tecnológicas para consulta, uso e adaptação por uma comunidade de usuários com propósitos não comerciais [Amiel et al., 2010]. Os REAs devem ser pensados como recursos educacionais que possam ser adaptados, mas também reutilizados em situações diferentes e alterados por outros usuários. Estes recursos devem estar sob domínio público ou licenciados de maneira aberta por licenças de direito autoral livre.

\subsection{Repositórios de Objetos de Aprendizagem}

Repositórios de Objetos de Aprendizagem (ROA) podem ser vistos como bancos de dados que armazenam Objetos de Aprendizagem e seus metadados. Os repositórios oferecem facilidades para localizar e obter objetos para serem usados em diversas situações de ensino e aprendizagem (Mohan, 2005). Um ROA também pode ser definido como um local na $W e b$ onde os objetos de aprendizagem são armazenados juntamente com seus metadados. Atualmente, a Web oferece vários repositórios específicos para armazenamento de objetos de aprendizagem. Porém, problemas relacionados à direitos autorais são comuns, o que tem forçado algumas instituições a criarem repositórios próprios com regras de comercialização bem claras.

\section{METODOLOGIA}

Nesta seção, apresentamos a metodologia adotada para a realização do estudo comparativo proposto neste trabalho. Inicialmente, foi feita a escolha dos repositórios e a definição dos critérios adotados na comparação e, em seguida, foi realizada a análise comparativa.

\footnotetext{
${ }^{1}$ https://okfn.org/
} 
Para a escolha dos repositórios, foram feitas pesquisas em sites de busca ${ }^{2}$, onde os seguintes termos foram usados como parâmetro: Repositório de Objetos de Aprendizagem e Learning Object Repository. Ao final desta etapa, chegamos a uma lista de 48 repositórios ${ }^{3}$ para serem analisados.

A escolha dos critérios para a comparação dos ROAs foi realizada a partir de uma análise das características dos OAs, dos Dados Abertos e dos REAs, tendo como ênfase o reúso dos conteúdos. Os critérios avaliados encontram-se no Quadro 3 e são detalhados a seguir.

\section{Quadro 3: Critérios para Análise dos ROAs}

\begin{tabular}{|c|l|l|}
\hline Número & \multicolumn{1}{|c|}{ Critério } & \multicolumn{1}{c|}{ Descrição } \\
\hline 1 & Livre acesso & $\begin{array}{l}\text { Os repositórios e os OAs disponibilizados por ele são de livre acesso para } \\
\text { qualquer usuário que quiser acessá-lo ? }\end{array}$ \\
\hline 2 & Formato disponibilizado & Os OAs são disponibilizados em formatos que facilitam o seu reúso ? \\
\hline 3 & Descrição de metadados & $\begin{array}{l}\text { Os OAs são disponibilizados com metadados que descrevem informações } \\
\text { relacionadas à identificação, conteúdo e histórico de um OA ? }\end{array}$ \\
\hline 4 & Fragmentação do conteúdo & Os OAs são disponibilizados por completo ou em fragmentos? \\
\hline 5 & Facilidade de consulta & A busca por OAs é fácil e objetiva ? \\
\hline 6 & Tipo de licença & A licença atribuída ao OA o deixe livre de qualquer restrição de uso ? \\
\hline
\end{tabular}

Durante a etapa de análise comparativa, cada um dos repositórios encontrados foi visitado e as tarefas apresentadas no Quadro 4 foram realizadas.

Quadro 4: Atividades realizadas para a análise comparativa

\begin{tabular}{|c|l|l|}
\hline Número & \multicolumn{1}{|c|}{ Critério } & \multicolumn{1}{c|}{ Atividade Realizada } \\
\hline 1 & Livre acesso & $\begin{array}{l}\text { Para cada um dos repositórios, o site correspondente foi acessado e uma consulta foi } \\
\text { realizada. Neste momento, verificamos se a busca por um OA era livre ou requeria algum } \\
\text { cadastro. }\end{array}$ \\
\hline 2 & $\begin{array}{l}\text { Formato } \\
\text { disponibilizado }\end{array}$ & $\begin{array}{l}\text { Para o OA encontrado, verificamos se o mesmo estava em um formato que facilitasse o seu } \\
\text { reúso, ou seja, se o OA estava disponível em um formato que pudesse ser facilmente } \\
\text { processado por máquina. }\end{array}$ \\
\hline 3 & $\begin{array}{l}\text { Descrição de } \\
\text { metadados }\end{array}$ & Para o OA encontrado, verificamos se há a descrição dos seus metadados. \\
\hline 4 & $\begin{array}{l}\text { Fragmentação } \\
\text { do conteúdo }\end{array}$ & $\begin{array}{l}\text { Para o OA encontrado foi verificado se estava disponível em fragmentos ou apenas por } \\
\text { completo. }\end{array}$ \\
\hline 5 & $\begin{array}{l}\text { Facilidade de } \\
\text { consulta }\end{array}$ & $\begin{array}{l}\text { Foram realizadas buscas por OAs a fim de verificar o quão fácil são as buscas no } \\
\text { repositório visitado. }\end{array}$ \\
\hline 6 & Tipo de licença & $\begin{array}{l}\text { Para cada repositório foi pesquisado no site informações sobre o tipo de licença atribuída } \\
\text { ao OA. }\end{array}$ \\
\hline
\end{tabular}

\section{RESULTADOS DA ANÁLISE COMPARATIVA}

O Quadro 5 sumariza os resultados obtidos a partir da análise comparativa dos repositórios de OAs.

Quadro 5: Resultados da análise comparativa

\section{Critério Atividade Realizada}

Livre acesso $\quad$ Verificou-se que em 6 repositórios, era preciso que o usuário, para ter acesso ao OA, fizesse um cadastro para se tornar usuário do repositório. Somente após o recebimento da confirmação de cadastro por email é que o usuário poderá retornar ao repositório, logar-se e ter acesso ao OA. Porém,

\footnotetext{
${ }^{2}$ http://scholar.google.com.br/http://link.springer.com/http://ieeexplore.ieee.org/Xplore/home.jsp http://www.scielo.org/php/index.php http://www.sciencedirect.com/

${ }^{3}$ http://profrossana.webnode.com/projeto-de-pesquisa/
} 


\begin{tabular}{|l|l|}
\hline & esse email para confirmação do cadastro as vezes não chega ou demora muito tempo para chegar. \\
\hline $\begin{array}{l}\text { Formato } \\
\text { disponibilizado }\end{array}$ & $\begin{array}{l}\text { Os principais formatos de OAs encontrados foram: .pdf, .ppt, .xlsx, .doc, .docx, .pptx, .txt, .xls,.pps e } \\
\text { ppsx. O mais comum é o .pdf, um formato que dificulta a reutilização do material, havendo a } \\
\text { necessidade de ferramentas específicas para a extração dos conteúdos e sua edição. }\end{array}$ \\
\hline $\begin{array}{l}\text { Descrição de } \\
\text { metadados }\end{array}$ & $\begin{array}{l}\text { Verificou-se que não há uma padronização dos metadados a serem preenchidos para cada tipo de OA. } \\
\text { Apenas 16 dos 48 repositórios avaliados solicitam alguma informação no momento do upload do OA. }\end{array}$ \\
\hline $\begin{array}{l}\text { Fragmentação } \\
\text { do conteúdo }\end{array}$ & $\begin{array}{l}\text { Ao fazer uma busca por OAs, foi detectado que nenhum dos repositórios oferece OAs de forma } \\
\text { fragmentada. Todos os OAs encontrados estavam por inteiro ou seja, um documento completo, um } \\
\text { capítulo completo de um livro, uma apresentação de um determinado assunto por inteiro. }\end{array}$ \\
\hline $\begin{array}{l}\text { Facilidade de } \\
\text { consulta }\end{array}$ & $\begin{array}{l}\text { Ao fazer uma busca por OAs, o usuário insere um termo para busca e são retornados OAs que } \\
\text { possuem informações relacionadas ao termo inserido. Porém, nem sempre este retorno traz somente } \\
\text { resultados esperados pelo usuário. }\end{array}$ \\
\hline Tipo de licença & $\begin{array}{l}\text { De uma maneira geral, os repositórios visitados não deixam claro que tipo de licença é atribuída para } \\
\text { cada OA disponibilizado. }\end{array}$ \\
\hline
\end{tabular}

\section{CONCLUSÕES}

Este trabalho apresentou o resultado de um estudo comparativo de alguns dos principais repositórios de OAs existentes. Como resultado da análise, verificou-se, que algumas características dos OAs, dos Dados Abertos e dos REAs, que podem contribuir para a reusabilidade dos OAs, ainda não são atendidas pelos repositórios existentes. Dentre estas características, destacamos a disponibilização em um formato que facilite sua reutilização e a disponibilização de OAs de forma fragmentada.

\section{REFERÊNCIAS}

AMIEL, Tel; OREY, Michael; WEST, Richard. (2010) "Recursos Educacionais Abertos (REA): modelos para localização e adaptação", In: ETD-Educação Temática Digital, v. 12, n. mar., p. 112-125.

MACEDO, L. N.; SALES, Gilvandenys Leite; CASTRO FILHO, José Aires de; OLIVEIRA, Eliana Moreira de; Macedo, SIQUEIRA, D. . (2007) "Desenvolvendo o pensamento proporcional com o uso de um objeto de aprendizagem". In: Carmem Lúcia Prata e Anna Christina Aun de Azevedo Nascimento. (Org.). Objetos de Aprendizagem - Uma Proposta de Recurso Pedagógico. Brasília: MEC/SEED, v.1, p. $-154$.

MOHAN, Permanand; KOOHANG, Alex; HARMAN, Keith. (2007) "Learning Objects Repositories”. In: Learning Objects: theory, praxis, issues, and trends. Santa Rosa: Informing Sciense Press.

SILVA, LAM; SANTANCHÈ, André. (2008) “Autoria de Objetos Digitais Complexos Baseada em Documentos Através da Anotação Semântica de Conteúdo", In: VII WTDBD-Workshop de Teses e Dissertações em Bancos de Dados, p. 25 - 30.

SILVA, M. da G. M. (2004) "Novas Aprendizagens", In: $11^{\circ}$ Congresso Internacional de Educação a Distância. Salvador - BA.

TAROUCO, L. M., \& DUTRA, R. (2007). "Padrões e interoperabilidade", In: Objetos de aprendizagem: uma proposta de recurso pedagógico. Brasília: MEC/SEED, p. 8192.

WILEY, D. A. (2000). "Learning object design and sequencing theory". Unpublished Ph.D. Thesis, Brigham Young University. In: http://davidwiley.com/papers/dissertation/dissertation.pdf Acesso em: 15/09/2014 